\title{
Fat Oxidation Kinetics Is Related to Muscle Deoxygenation Kinetics During Exercise
}

\begin{abstract}
Anouck Zurbuchen ${ }^{1}$, Stefano Lanzi ${ }^{2,3}$, Ludovic Voirol ${ }^{2}$, Cybele Barboza Trindade ${ }^{2}$, Boris Gojanovic ${ }^{4}$, Bengt Kayser ${ }^{2}$, Nicolas Bourdillon ${ }^{2}$, Xavier Chenevière ${ }^{1 * t}$ and Davide Malatesta ${ }^{2 \dagger}$
\end{abstract}

\begin{abstract}
'Department of Neurosciences and Movement Science, Faculty of Science and Medicine, University of Fribourg, Fribourg, Switzerland, ${ }^{2}$ Institute of Sport Sciences of the University of Lausanne, Doctrine Selon Convention SSP-FBM, University of Lausanne, Lausanne, Switzerland, ${ }^{3}$ Division of Angiology, Heart and Vessel Department, Lausanne University Hospital, Lausanne, Switzerland, ${ }^{4}$ Sports Medicine Unit, Swiss Olympic Medical Center, Department for Locomotion, Lausanne University Hospital, Lausanne, Switzerland
\end{abstract}

Purpose: The present study aimed to determine whether whole-body fat oxidation and muscle deoxygenation kinetics parameters during exercise were related in individuals with different aerobic fitness levels.

OPEN ACCESS

Edited by:

François Billaut,

Laval University, Canada

Reviewed by:

Thomas Jackson Barstow, Kansas State University, United States Juan Manuel Murias,

University of Calgary, Canada

*Correspondence: Xavier Chenevière

xavier.cheneviere@unifr.ch

${ }^{\dagger}$ These authors have contributed equally to this work

Specialty section: This article was submitted to

Exercise Physiology, a section of the journa

Frontiers in Physiology

Received: 23 January 2020 Accepted: 07 May 2020 Published: 04 June 2020

Citation: Zurbuchen A, Lanzi S, Voirol L, Trindade CB, Gojanovic B, Kayser B, Bourdillon N, Chenevière $X$ and Malatesta D (2020) Fat Oxidation Kinetics Is Related to Muscle

Deoxygenation Kinetics During Exercise. Front. Physiol. 11:571. doi: 10.3389/fphys.2020.00571
Methods: Eleven cyclists [peak oxygen uptake $\left(\dot{V} O_{2 \text { peak }}\right): 64.9 \pm 3.9 \mathrm{~mL} \cdot \mathrm{kg}^{-1} \cdot \mathrm{min}^{-1}$ ] and 11 active individuals $\left(\dot{V} O_{2 p e a k}: 49.1 \pm 7.4 \mathrm{~mL} \cdot \mathrm{kg}^{-1} \cdot \mathrm{min}^{-1}\right)$ performed a maximal incremental cycling test to determine $\dot{V} \mathrm{O}_{2 p e a k}$ and a submaximal incremental cycling test to assess whole-body fat oxidation using indirect calorimetry and muscle deoxygenation kinetics of the vastus lateralis (VL) using near-infrared spectroscopy (NIRS). A sinusoidal (SIN) model was used to characterize fat oxidation kinetics and to determine the intensity (Fat max) eliciting maximal fat oxidation (MFO). The muscle deoxygenation response was fitted with a double linear model. The slope of the first parts of the kinetics $\left(a_{1}\right)$ and the breakpoint $\left([\mathrm{HHb}]_{\mathrm{BP}}\right)$ were determined.

Results: MFO $(p=0.01)$ and absolute fat oxidation rates between 20 and $65 \%$ $\dot{V} O_{2 p e a k}$ were higher in cyclists than in active participants $(p<0.05)$, while Fat max $_{\text {max }}$ occurred at a higher absolute exercise intensity $(p=0.01) . a_{1}$ was lower in cyclists $(p=0.02)$ and $[\mathrm{HHb}]_{\mathrm{BP}}$ occurred at a higher absolute intensity $(p<0.001)$ than in active individuals. $\dot{V} \mathrm{O}_{2 \text { peak }}$ was strongly correlated with MFO, Fat max, and $[\mathrm{HHb}]_{\mathrm{BP}}(r=0.65$ $0.88, p \leq 0.001)$. MFO and Fat ${ }_{\max }$ were both correlated with $[\mathrm{HHb}]_{\mathrm{BP}}(r=0.66, p=0.01$ and $r=0.68, p<0.001$, respectively) and tended to be negatively correlated with $a_{1}$ ( $r=-0.41, p=0.06$ for both).

Conclusion: This study showed that whole-body fat oxidation and muscle deoxygenation kinetics were both related to aerobic fitness and that a relationship between the two kinetics exists. Individuals with greater aerobic fitness may have a delayed reliance on glycolytic metabolism at higher exercise intensities because of a longer maintained balance between $\mathrm{O}_{2}$ delivery and consumption supporting higher fat oxidation rates.

Keywords: aerobic fitness, cycling, indirect calorimetry, Fat ${ }_{\max }$, NIRS, breaking point 


\section{INTRODUCTION}

Regular endurance training leads to development of the muscle capillary network and marked changes in mitochondrial content and activity or in muscle fiber type composition (see Hawley et al., 2014 for review). Increased skeletal muscle capillarization enhances the substrate-exchange surface and fatty acid entry into myocytes (Sahlin et al., 2008). This leads to a greater fat oxidation capacity (Stisen et al., 2006; Sahlin et al., 2008) and delayed reliance on carbohydrates (Brooks and Mercier, 1994), resulting in a "glycogen-sparing effect" (Holloszy and Coyle, 1984) in endurance-trained athletes. The fat oxidation capacity may be a determining factor in endurance performance (Frandsen et al., 2017).

The whole-body fat oxidation rate measured during the incremental exercise test allows the estimation of an individual's ability to oxidize fat (Achten et al., 2002; Cheneviere et al., 2009). Fat oxidation kinetics expressed as a function of exercise intensity gives a parabolic curve, with the maximal fat oxidation (MFO) rate occurring at an intensity defined as "Fat ${ }_{\max }$ " (Achten et al., 2002). Achten and Jeukendrup (2003) showed a greater fat oxidation rate over a wide range of intensities and a higher $\mathrm{MFO}$ in athletes with maximal oxygen uptake $\left(\dot{V} \mathrm{O}_{2 p e a k}\right)$ above $65 \mathrm{~mL} \cdot \mathrm{kg}^{-1} \cdot \mathrm{min}^{-1}$ compared to athletes with values below this level. This was concomitant with similar relative $\mathrm{Fat}_{\max }$ values expressed as $\% \dot{V} O_{2 p e a k}$ in both groups. Similar results were found when comparing trained with untrained men (LimaSilva et al., 2010). In addition to MFO and $\mathrm{Fat}_{\max }$, Cheneviere et al. (2009) developed a sinusoidal (SIN) model with three independent variables (dilatation, symmetry, and translation) to mathematically characterize the shape of the whole-body fat oxidation kinetics as a function of exercise intensity (see Figure 1 for a graphic representation of these three variables). As aerobic fitness influences fat oxidation capacity, the greater MFO and fat oxidation rates across exercise intensities found in trained athletes (Achten and Jeukendrup, 2003; Stisen et al., 2006; Lima-Silva et al., 2010) may result in an upward and more dilated (greater dilatation) projection of the fat oxidation kinetics compared to less-trained individuals.

A better oxygen $\left(\mathrm{O}_{2}\right)$ delivery and utilization into mitochondria may partly explain the greater fat oxidation capacity in endurance-trained athletes. In fact, endurance training leads to improved muscle blood perfusion. The increased muscular blood flow $\left(\dot{Q}_{\mathrm{m}}\right)$ provides more $\mathrm{O}_{2}$ to the working muscles, allowing a better balance between $\mathrm{O}_{2}$ delivery and muscular $\mathrm{O}_{2}$ utilization $\left(\dot{V}_{2 \mathrm{~m}}\right)$ (Koga et al., 2014). Given the exclusive reliance on $\mathrm{O}_{2}$ for fat use as an energy substrate, it would be expected that differences in fat oxidation capacity would be mirrored by differences in the regulation of muscle $\mathrm{O}_{2}$ supply. The $\dot{Q}_{\mathrm{m}} / \dot{V} \mathrm{O}_{2 \mathrm{~m}}$ ratio is considered an important determinant of aerobic performance and seems to be linked to the aerobic capacity (Ferreira et al., 2007; Boone et al., 2012). This ratio can be estimated by integrating the near-infrared spectroscopy (NIRS), a non-invasive technique used to assess tissue oxygenation levels and changes during exercise (see Grassi and Quaresima, 2016 for review), with the pulmonary $\dot{V} O_{2}$ data. NIRS measures variations in the deoxygenated hemo- and myoglobin concentration $(\Delta[\mathrm{HHb}]) . \Delta[\mathrm{HHb}]$ is considered to be an indicator of the balance between $\mathrm{O}_{2}$ delivery and consumption, providing a solid estimation of the dynamic profile of $\mathrm{O}_{2}$ extraction within the area of NIRS interrogation (DeLorey et al., 2003). Some authors acknowledge that the dynamic relationship between $\dot{Q}_{\mathrm{m}}$ and $\dot{V} \mathrm{O}_{2 \mathrm{~m}}$ follows a sigmoid model during incremental exercise: $\dot{Q}_{\mathrm{m}}$ kinetics is initially faster than $\dot{V} O_{2 m}$ kinetics and slows progressively to approximate $\dot{V} \mathrm{O}_{2 \mathrm{~m}}$ kinetics as the exercise intensity increases (Ferreira et al., 2007; Boone et al., 2009). However, it seems that the $\Delta[\mathrm{HHb}]$ response may be better described by a double linear model (Spencer et al., 2012) because it would more precisely explain the physiological mechanisms underlying the $\Delta[\mathrm{HHb}]$ kinetics (see Boone et al., 2016b for review). According to this model, $\Delta[\mathrm{HHb}]$ increases linearly with work load until a breakpoint $\left([\mathrm{HHb}]_{\mathrm{BP}}\right)$, followed by a near-plateau. Although somewhat controversial (Broxterman et al., 2018; Keir et al., 2018), [HHb] $]_{\text {BP }}$ potentially reflects a physiological response, such as maximal lactate steady state, critical power or the respiratory compensation point (RCP). Since endurance athletes have good oxidative capacity, their $[\mathrm{HHb}]_{\text {BP }}$ may appear at higher absolute (McNarry et al., 2015) and relative intensities (Boone et al., 2009, 2016a; Murias et al., 2013), reflecting delayed reliance on anaerobic ATP production. Therefore, the first part of the muscular deoxygenation kinetics may present a rightward shift and a slower slope in endurance athletes who characteristically have a higher aerobic capacity compared to sedentary individuals (Boone et al., 2016a).

The adaptations to endurance training affecting aerobic fitness seem to influence both muscular deoxygenation and fat oxidation kinetics. However, to the best of our knowledge, the relationship between the two kinetics has never been investigated in healthy individuals of various aerobic fitness levels. Therefore, the purpose of the present study was to determine whether wholebody fat oxidation and muscle deoxygenation kinetics parameters during exercise were related in individuals with different aerobic fitness levels (trained cyclists versus active individuals). It was hypothesized that (i) cyclists would have shallower muscular deoxygenation kinetics with a right-shifted $[\mathrm{HHb}]_{\mathrm{BP}}$, as well as upward and more dilated (greater dilatation) fat oxidation kinetics and (ii) a direct relationship between both kinetics would exist, with shallower muscular deoxygenation kinetics corresponding to higher fat oxidation rates.

\section{MATERIALS AND METHODS}

\section{Participants}

Twenty-two healthy male subjects [11 daily trained cyclists and triathletes $\left(\dot{V} O_{2 \text { peak }} \geq 60 \mathrm{~mL} \cdot \mathrm{kg}^{-1} \cdot \mathrm{min}^{-1}\right)$ and 11 nonspecifically trained but active individuals] aged between 20 and 39 years participated in this study (Table 1). They declared that they were non-smokers, disease-free, and not taking any medication. All subjects were fully informed about the protocol and its possible risks and benefits, and they all provided written consent. The protocol was conducted according to the Declaration of Helsinki and was approved by the local ethics 
TABLE 1 | Participant physical characteristics and aerobic fitness parameters.

\begin{tabular}{|c|c|c|}
\hline & Cyclists $(n=11)$ & Active group $(n=11)$ \\
\hline \multicolumn{3}{|l|}{ Participant characteristics } \\
\hline Age [years] & $27.4 \pm 5.1$ & $28.7 \pm 5.1$ \\
\hline Height [m] & $1.83 \pm 0.03$ & $1.80 \pm 0.07$ \\
\hline Weight [kg] & $72.3 \pm 2.8$ & $77.7 \pm 8.1^{*}$ \\
\hline $\mathrm{BMI}\left[\mathrm{kg} \cdot \mathrm{m}^{-2}\right]$ & $21.7 \pm 0.9$ & $23.9 \pm 2.4^{\star}$ \\
\hline FFM [kg] & $64.2 \pm 2.7$ & $65.7 \pm 5.1$ \\
\hline $\mathrm{FM}[\mathrm{kg}]$ & $8.1 \pm 2.9$ & $12.0 \pm 4.0^{*}$ \\
\hline FM [\%] & $11.1 \pm 3.7$ & $15.2 \pm 3.8^{\star}$ \\
\hline \multicolumn{3}{|l|}{ Maximal exercise test } \\
\hline MAP $\left[\mathrm{W} \cdot \mathrm{kg}^{-1}\right]$ & $5.5 \pm 0.4$ & $4.1 \pm 0.6^{\star}$ \\
\hline$\dot{V} O_{2 p e a k}\left[\mathrm{~mL} \cdot \mathrm{kg}^{-1} \cdot \mathrm{min}^{-1}\right]$ & $64.9 \pm 3.9$ & $49.1 \pm 7.4^{\star}$ \\
\hline $\mathrm{RCP}\left[\mathrm{mL} \cdot \mathrm{kg}^{-1} \cdot \mathrm{min}^{-1}\right]$ & $56.1 \pm 4.2$ & $41.7 \pm 7.4^{*}$ \\
\hline $\mathrm{RCP}\left[\% \dot{V} \mathrm{O}_{2 \text { peak }}\right]$ & $86.4 \pm 2.1$ & $84.8 \pm 5.4$ \\
\hline $\mathrm{VT}\left[\mathrm{mL} \cdot \mathrm{kg}^{-1} \cdot \mathrm{min}^{-1}\right]$ & $43.1 \pm 4.5$ & $30.4 \pm 7.0^{*}$ \\
\hline $\mathrm{VT}\left[\% \dot{V} O_{2 p e a k}\right]$ & $66.4 \pm 4.7$ & $61.5 \pm 7.7$ \\
\hline
\end{tabular}

Data are presented as the mean $\pm S D$. BMI, body mass index; FM, fat mass; FFM, fat-free mass; MAP, maximal aerobic power; $\dot{V} O_{2 p e a k}$, peak oxygen uptake; VT, ventilatory threshold; $R C P$, respiratory compensation point. *Significant difference between groups $(p \leq 0.05)$.

committee (Cantonal Swiss Ethics Committees on research involving humans).

\section{Experimental Design}

Each subject completed three test sessions. In the first session, anthropometric measurements (i.e., height, body mass, and composition) were taken. Each subject then performed a maximal incremental exercise test on a cycle ergometer. For the remaining two sessions, the subjects performed two identical submaximal incremental exercise tests [i.e., until a respiratory exchange ratio (RER) of 1.0]. Sessions 2 and 3 were identical and separated by a minimum of 7 days and a maximum of 10 days. The pre-experimental conditions were controlled to standardize measurements and to minimize inter- and intra-individual variations. Both tests were performed in the morning after a minimum 10-h overnight fast, after 3 days of a balanced diet (2300-2500 kcal/day), with no caffeine and no alcohol during the last $48 \mathrm{~h}$, and the volunteers were asked to refrain from vigorous exercise during the day before the experimental trials. Before the beginning of the testing session, all the subjects confirmed that they had followed the nutritional indications, and the food diary was checked by the experimenters.

\section{Assessments}

\section{Anthropometric Measurements}

Height, body mass, and composition were assessed. Body composition [i.e., fat mass (FM) and lean mass] was estimated from the Durnin and Womersley's skinfold-thickness measurement method at four sites (Durnin and Womersley, 1973).

\section{Aerobic Fitness}

To determine peak oxygen uptake $\left(\dot{V} O_{2 p e a k}\right)$, maximal aerobic power (MAP), ventilatory threshold (VT), and RCP, each subject performed an incremental exercise test to exhaustion on a cycle ergometer (Ebike Basic BPlus, General Electric, Niskayuna, NY, United States). After a 3-min rest on the ergometer, the test began with a 5-min warm-up at $60 \mathrm{~W}$. The power output was then increased by $30 \mathrm{~W}$ every minute until exhaustion (Malatesta et al., 2009). Pedaling frequency was maintained between 70 and 90 revolutions per minute (rpm). The maximal incremental test ended when participants failed to maintain a pedaling frequency $>60 \mathrm{rpm}$ despite maximum effort and verbal encouragement. Gas exchanges were continuously measured with a breath-by-breath online system (OxyconPro, Jaeger, Germany). The device was calibrated in three steps before each test: (1) ambient air analysis; (2) gas analyzer calibration with a known gas mixture $\left(16.00 \% \mathrm{O}_{2}, 5.02 \% \mathrm{CO}_{2}\right)$; and (3) volume turbine calibration with a 3 -L syringe. $\dot{V} \mathrm{O}_{2} \dot{\mathrm{V}} \mathrm{O}_{2 \text { peak }}$ corresponded to the highest $\dot{V} \mathrm{O}_{2}$ value computed from a 15-s rolling average during the test. The power output corresponding to $\dot{V} O_{2 p e a k}$ or to the lowest power output corresponding to the plateau of $\dot{V} \mathrm{O}_{2}$ (i.e., a plateau of $\dot{V} \mathrm{O}_{2}$ corresponded to an increase of no more than $2 \mathrm{~mL} \cdot \mathrm{kg}^{-1} \cdot \mathrm{min}^{-1}$ with an increase of workload during the latter stages of the test) was considered as the MAP. The VT and RCP were estimated by three blinded and independent investigators: ventilatory equivalent method was used to determine both thresholds (Wasserman et al., 1973), while V-slope method was used to confirm VT (Beaver et al., 1986).

\section{Whole-Body Fat Oxidation Kinetics}

Both submaximal experimental trials served to determine wholebody fat oxidation kinetics. Resting values were measured during a 10 -min seated-rest. This was followed by a 10-min warm-up at $20 \%$ MAP. The workload was then increased by $7.5 \%$ MAP for the active group and 10\% MAP for cyclists every 5 min until the end of the stage during which RER was 1.0 (Cheneviere et al., 2011). For the submaximal exercise test, pedaling frequency was maintained between 70 and $90 \mathrm{rpm}$. Average values for $\dot{V} \mathrm{O}_{2}$ and $\dot{V} \mathrm{CO}_{2}$ were calculated during the last minute of warm-up and during the last minute of each stage of the test. The values obtained during each test session were then ensemble-averaged to reduce the intra-individual variability of fat oxidation rates using indirect calorimetry (Croci et al., 2014). Fat oxidation rates were calculated using stoichiometric equations (Frayn, 1983). The results of the submaximal incremental tests were used to calculate the fat oxidation rates over a wide range of exercise intensities. To model whole-body fat oxidation kinetics, represented as a function of exercise intensity, and to determine Fat max $_{\text {and }}$ MFO, the SIN model was used (Cheneviere et al., 2009). This model includes three independent variables, representing the main quantitative characteristics of the curve, namely, dilatation, symmetry, and translation, indicated as $d, s$, and $t$, respectively, as well as pi $(\pi)$ corresponding to $\sim 3.14$ and a constant of intensity named $K$, which corresponds to $(\pi / 100)$ :

$$
\% M F O=\sin \left[\left\{\frac{\pi^{1 / s}}{\pi+2 d}\left(K \cdot \% \dot{V} O_{2 p e a k}+d+t\right)\right\}^{s}\right]
$$


where dilatation refers to the degree of dilation or retraction of the curve, symmetry modifies the symmetry of the standard basic sine curve, and translation refers to the right-left shift of the whole curve toward the abscissa axis (Figure 1). To fit the fat oxidation rates and to model the fat oxidation kinetics, the three variables were independently changed using an iterative procedure by minimizing the sum of the mean squares (SMS) of the differences between the estimated energy derived from lipid ( $E_{\text {lipid }}$ ) based on the SIN model and the calculated values of $E_{\text {lipid }}$, as described in a previous study (Cheneviere et al., 2009). For each subject, Fat max $_{\max }$ was calculated by differentiation of the SIN model equation, and the fat oxidation rate was determined at every $5 \% \dot{V} O_{2 \text { peak }}$ between 20 and $85 \% \dot{V} O_{2 \text { peak }}$.

\section{Muscle Deoxygenation Kinetics}

During both submaximal exercise tests, deoxyhemoglobin $(\mathrm{HHb})$, oxyhemoglobin $\left(\mathrm{O}_{2} \mathrm{Hb}\right)$, and total hemoglobin $\left(\mathrm{Hb}_{\text {tot }}\right)$ concentrations were continuously measured in the right vastus lateralis (VL) muscle using NIRS (Oxymon Mk III, Artinis Medical System, Netherlands) at $50 \mathrm{~Hz}$ to determine muscle deoxygenation. The NIRS probe contains a three-diode emitter operating at wavelengths of 764 and $860 \mathrm{~nm}$ and a detector positioned $40 \mathrm{~mm}$ away from the emitter. After calibration of the device, the probe was positioned on shaved and cleaned skin over the right VL muscle, halfway between the great trochanter and lateral femoral condyle, parallel to the major axis of the thigh. The VL muscle was chosen to assess exerciseinduced changes in $[\mathrm{HHb}]$ concentrations since this muscle is highly active during cycling (Laplaud et al., 2006). After this placement, pen marks were made on the skin at the margins of the probe to verify that it did not move during the trial and to enable the exact same placement in the next test session. The probe was then firmly fixed to the thigh with tape, a bandage, and the aid of the subject's shorts, reducing the influence of ambient light on the measurements. Light absorption changes are converted into $[\mathrm{HHb}$ ] concentration changes using a modified Lambert-Beer law in which a path-length factor is incorporated to correct for the scattering of photons in the tissue (Ferrari et al., 1997). A differential path-length factor of 6.5 was used (Duncan et al., 1995). Changes in [HHb] concentrations were downsampled to $1 \mathrm{~Hz}$ and then time aligned to the onset of exercise and ensemble-averaged. Average values for [HHb] were calculated during the last minute of warm-up and during the last minute of every stage of the incremental submaximal test. The values obtained were then normalized so that $0 \%$ represented the steady-state $[\mathrm{HHb}]$ value during warm-up and $100 \%$ represented the highest average [HHb] value observed in the exercise test (i.e., $\% \Delta[\mathrm{HHb}]$ ). The normalized values were plotted as a function of absolute $\left(\dot{V} \mathrm{O}_{2}\right)$ and relative exercise intensity $\left(\% \dot{V} O_{2 p e a k}\right)$ and were analyzed by means of a double linear model (Spencer et al., 2012; Boone et al., 2016a) as follows:

$\% \Delta[\mathrm{HHb}]= \begin{cases}a_{1} \cdot x+b & \text { if } x \leq[H H b]_{\mathrm{BP}} \\ a_{1} \cdot x+a_{2}(x-B P)+b & \text { if } x>[H H b]_{\mathrm{BP}}\end{cases}$ where $a_{1}$ is the slope of the linear regression before the breaking point $\left([\mathrm{HHb}]_{\mathrm{BP}}\right) ; b$ is the $y$-intercept of the linear regression before the $[\mathrm{HHb}]_{\mathrm{BP}}$; and $a_{2}$ is the change in the slope of the linear regression after the $[\mathrm{HHb}]_{\mathrm{BP}}$. The model parameters were estimated using an iterative procedure by minimizing the SMS of the differences between the estimated $\% \Delta[\mathrm{HHb}]$ based on the double linear model and the measured values of $\% \Delta[\mathrm{HHb}]$. The slope of the linear regression after the $[\mathrm{HHb}]_{\mathrm{BP}}\left(a_{3}\right)$ was then calculated as the sum of $a_{1}$ and $a_{2}$.

\section{Statistical Analysis}

Data are expressed as the mean \pm SD for all variables. The sample size of 11 participants per group was calculated based on previously published data (Nordby et al., 2006). Before each statistical test, the normality and equality of variances of the data were verified using the Shapiro-Wilk test and Levene test, respectively. Differences in physical characteristics, aerobic fitness, SIN model, and double linear parameters between trained cyclists and active individuals were assessed using a twosamples $t$-test or the Mann-Whitney test for non-parametric data. Independent correlations between the parameters of wholebody fat oxidation kinetics and those of the double linear model of $\% \Delta[\mathrm{HHb}]$ were performed by using Pearson $(r)$ or Spearman (rho) correlation coefficients. Furthermore, a two-way repeatedmeasures mixed design ANOVA (group $\times$ intensity) was used to compare the change in fat oxidation rates with exercise intensity (in a range from 20 to $85 \%$ of $\dot{V} O_{2 p e a k}$ ) between both groups. The level of significance was set at $p \leq 0.05$. Data analyses were all performed using RStudio (version 0.98.1103).

\section{RESULTS}

\section{Participants}

The participants' physical characteristics and their aerobic fitness parameters are listed in Table 1. The age and height were similar in both groups ( $p=0.40$ and $p=0.36$, respectively). In comparison with the active group, cyclists had a lower body mass index (BMI) $(p=0.02)$ and FM (in $\%$ and in $\mathrm{kg})(p=0.02$ for both), while their $\dot{V} O_{2 p e a k}$ and MAP were higher $(p<0.001)$, confirming that the aerobic fitness level was different between the two experimental groups. Although no difference was found in the RCP and VT expressed as $\% \dot{V} O_{2 p e a k}$ between the two groups ( $p=0.40$ and $p=0.09$, respectively), the absolute values of the VT and RCP were higher in cyclists $(p<0.001)$.

\section{Whole-Body Fat Oxidation Kinetics}

Whole-body fat oxidation kinetics, expressed in relative and absolute values, are shown in Figures $2 \mathrm{~A}-\mathrm{C}$, respectively. The absolute fat oxidation rate $\left(\mathrm{mg} \cdot \mathrm{kg}^{-1} \cdot \mathrm{min}^{-1}\right)$ was higher in the cyclist group $(p=0.03)$ than that in the active group for the exercise intensities between 20 and $65 \% \dot{V} O_{2 p e a k}$ $(p \leq 0.046$; Figure 2C). Table 2 shows the whole-body fat oxidation parameters and SIN model variables. MFO and Fat $_{\text {max }}$ in $\mathrm{mL} \cdot \mathrm{kg}^{-1} \cdot \mathrm{min}^{-1}$ were higher in cyclists than in active individuals ( $p=0.01$ for both). However, no difference was found when $\mathrm{Fat}_{\max }$ was expressed as $\% \dot{V} O_{2 p e a k}(p=0.77)$. 

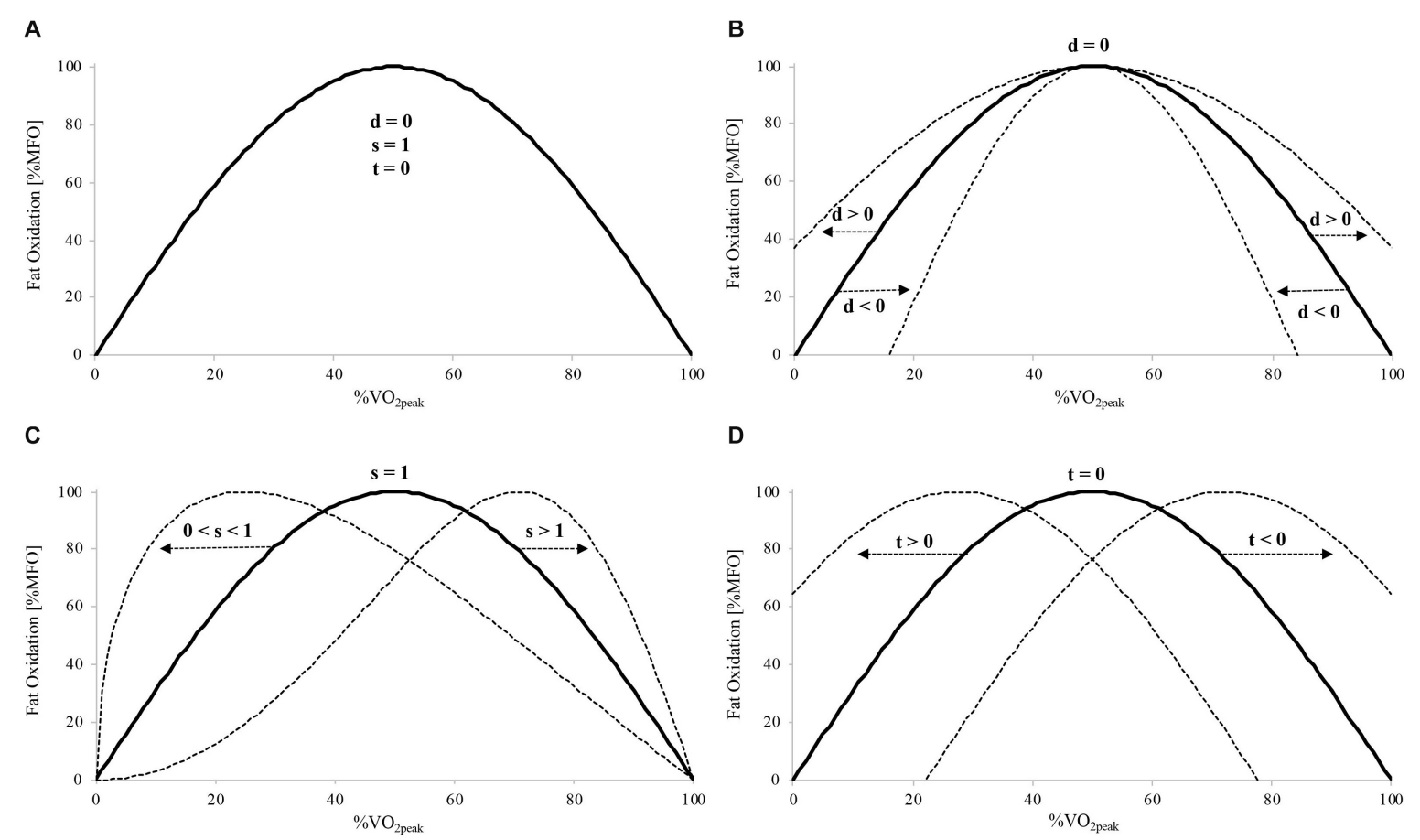

FIGURE 1 | Schematic illustration of the three independent variables of the SIN model (dilatation [d], symmetry [s], and translation [t]) and their impact on the whole-body fat oxidation kinetics. Basic symmetric $\mathrm{SIN}$ curve with $\mathrm{d}=0, \mathrm{~s}=1$, and $\mathrm{t}=0$ (A). Changes in $\mathrm{d}(\mathbf{B}), \mathrm{s}$ (C), and $\mathrm{t}$ (D) and corresponding modifications in the basic symmetric SIN curve (dotted lines). MFO, maximal fat oxidation; $\dot{V} O_{2 p e a k}$, peak oxygen uptake.

The dilatation was greater in the active group than in the cyclist group $(p=0.003)$, while no difference in symmetry or translation was found between the two groups $(p=0.92$ and $p=0.64$, respectively).

Maximal fat oxidation was positively correlated with $\dot{V} O_{2 p e a k}$ $(r=0.65 ; p=0.001)$, MAP ( $r h o=0.43 ; p=0.045)$, and VT in $\mathrm{mL} \cdot \mathrm{kg}^{-1} \cdot \mathrm{min}^{-1}(r=0.74 ; p<0.001)$ and in $\% \dot{V} O_{2 p e a k}(r=0.68$; $p=0.001)$ and was correlated with RCP only when expressed in $\mathrm{mL} \cdot \mathrm{kg}^{-1} \cdot \mathrm{min}^{-1}(r=0.62 ; p=0.002)$. Similarly, Fat $\max$ $\left(\mathrm{mL} \cdot \mathrm{kg}^{-1} \cdot \mathrm{min}^{-1}\right)$ was also positively correlated with $\dot{V} O_{2 p e a k}$ $(r=0.73 ; p<0.001)$ and $\mathrm{VT}$ in $\mathrm{mL} \cdot \mathrm{kg}^{-1} \cdot \mathrm{min}^{-1}(r=0.77$; $p<0.001)$ and in $\% \dot{V} O_{2 p e a k}(r=0.59 ; p=0.004)$ and was correlated with RCP only when expressed in $\mathrm{mL} \cdot \mathrm{kg}^{-1} \cdot \mathrm{min}^{-1}$ $(r=0.69 ; p<0.001)$. In contrast, the relative value of Fat ${ }_{\max }$ was not correlated with any aerobic fitness parameters.

\section{Muscle Deoxygenation Kinetics}

The $\% \Delta[\mathrm{HHb}]$ response as a function of relative and absolute exercise intensity is shown in Figures 2D,E, respectively. The parameters of the double linear model as a function of relative exercise intensity for the two experimental groups are presented in Table 3. These values indicate that the curve of muscle deoxygenation kinetics had a shallower slope with a right-shifted $[\mathrm{HHb}]_{\mathrm{BP}}$ in cyclists compared to that in active individuals. In fact, $a_{1}$ was lower in the cyclist group than in the active group $(p=0.02)$. The $[\mathrm{HHb}]_{\mathrm{BP}}$ occurred at a higher absolute intensity (in $\mathrm{mL} \cdot \mathrm{kg}^{-1} \cdot \mathrm{min}^{-1}$ ) but not at a higher relative intensity (in $\% \dot{V} O_{2 p e a k}$ ) in cyclists than in active individuals ( $p<0.001$ and $p=0.098$, respectively). The $[\mathrm{HHb}]_{\mathrm{BP}}$ expressed in $\mathrm{mL} \cdot \mathrm{kg}^{-1} \cdot \mathrm{min}^{-1}$ was strongly and positively correlated with $\dot{V} O_{2 p e a k}(r=0.88 ; p<0.001)$, MAP $(r h o=0.80 ; p<0.001)$, VT (in $\mathrm{mL} \cdot \mathrm{kg}^{-1} \cdot \mathrm{min}^{-1}$ and in $\% \dot{V} O_{2 p e a k}: r=0.83 ; p<0.001$ and $r=0.50 ; p=0.02$, respectively), and $\mathrm{RCP}$ (in $\mathrm{mL} \cdot \mathrm{kg}^{-1} \cdot \mathrm{min}^{-1}$, $r=0.87 ; p<0.001)$.

\section{Correlations Between Fat Oxidation and Muscle Deoxygenation Kinetics}

The correlations between whole-body fat oxidation and muscle deoxygenation parameters are listed in Table 4. MFO tended to be negatively correlated with $a_{1}(p=0.06)$ and was positively correlated with $[\mathrm{HHb}]_{\mathrm{BP}}(p=0.01)$. Fat $\max \left(\mathrm{mL} \cdot \mathrm{kg}^{-1} \cdot \mathrm{min}^{-1}\right)$ tended to be negatively correlated with $a_{1}(p=0.06)$ and was positively correlated with $a_{3}(p=0.03)$, [HHb] $]_{\mathrm{BP}}(p<0.001)$. When expressed in relative values $\left(\% \dot{V} O_{2 p e a k}\right)$, Fat ${ }_{\text {max }}$ was only positively correlated with $a_{3}(p=0.01)$. Finally, the symmetry was positively correlated with $a_{3}(p=0.01)$. A schematic view of the correlations among aerobic fitness, whole-body fat oxidation and muscle deoxygenation kinetics is presented in Figure 3.

\section{DISCUSSION}

The present study showed that the whole-body fat oxidation and muscle deoxygenation kinetics during exercise were related in individuals with different aerobic fitness levels. Cyclists 

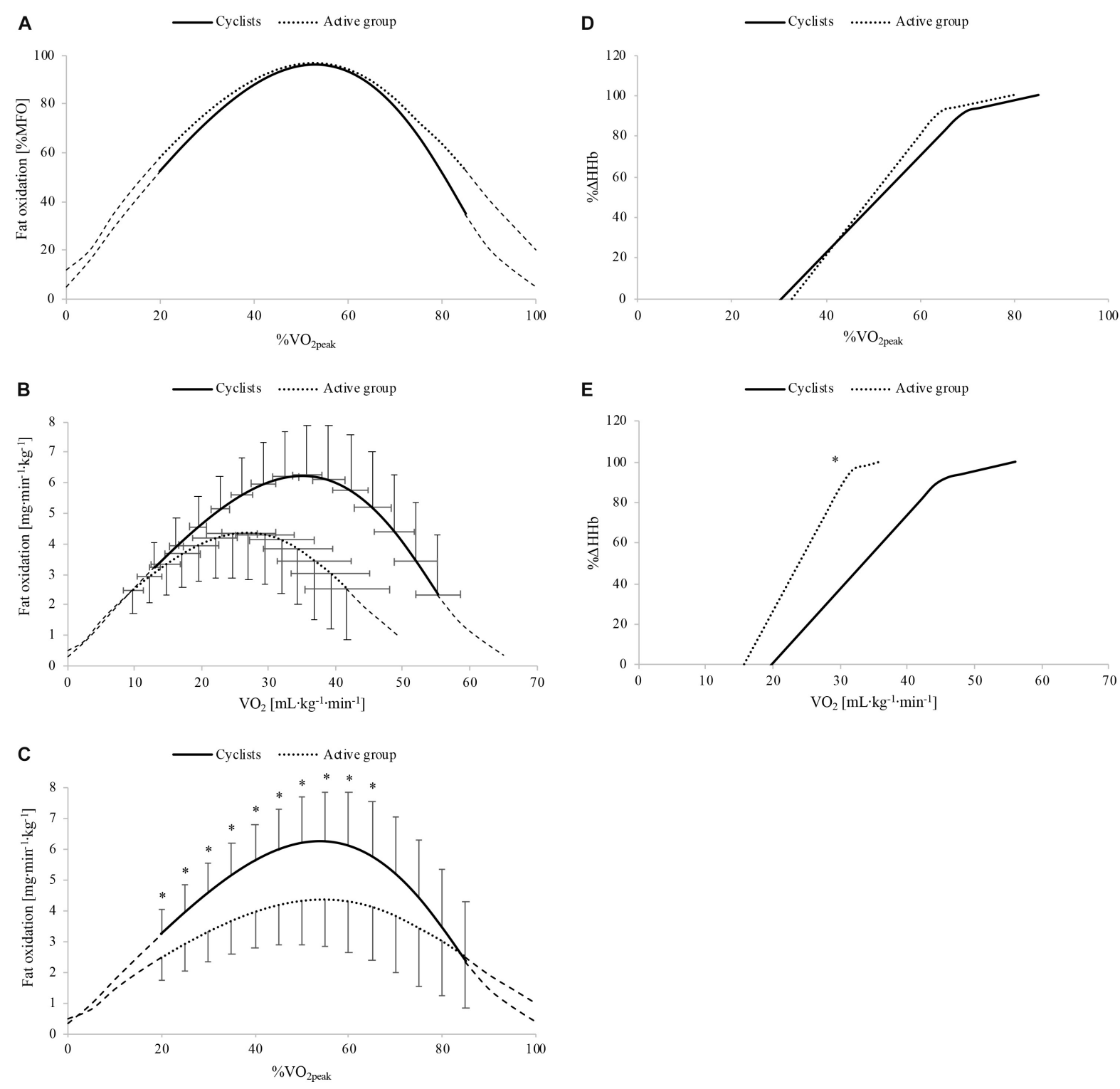

FIGURE 2 | Whole-body fat oxidation kinetics in relative (A) and absolute (B,C) values and muscle deoxygenation kinetics as a function of relative (D) and absolute (E) exercise intensity in cyclists (black line) and active people (black dots). $\Delta[\mathrm{HHb}]$, variation in deoxygenated hemo- and myoglobin concentration; MFO, maximal fat oxidation; $\dot{V} O_{2 p e a k}$, peak oxygen uptake. *Significant difference between the cyclist and active group $(p \leq 0.05)$.

exhibited (1) significant upward fat oxidation kinetics with greater MFO and absolute Fat $\max _{\max }$ and (2) shallower muscular deoxygenation kinetics (i.e., lower $a_{1}$ values) with a right-shifted $[\mathrm{HHb}]_{\mathrm{BP}}$ compared to active participants. MFO and Fat $\max _{\max }$ were both positively correlated with the $[\mathrm{HHb}]_{\mathrm{BP}}$ and tended to be negatively correlated with $a_{1}$ (Table 4 and Figure 3).

\section{Effect of Aerobic Fitness on Whole-Body Fat Oxidation Kinetics}

Whole-body fat oxidation rates for intensities from 20 to $65 \%$ $\dot{V} O_{2 \text { peak }}$ (Figure 2C) and MFO (Table 2) were greater (+39\%) in cyclists than in the active group, corroborating previous studies comparing trained and very trained men (Achten and Jeukendrup, 2003; Lima-Silva et al., 2010) or trained and untrained women (Stisen et al., 2006). Furthermore, consistent with these studies, MFO was positively correlated with $\dot{V} O_{2 p e a k}$, indicating an increased fat oxidation capacity in endurancetrained athletes. This may be due to several endurance-training adaptations, such as an increased mitochondrial volume and activity (Holloszy and Coyle, 1984; Bishop et al., 2014), an enhanced oxidative enzyme activity (Stisen et al., 2006), or a greater amount of oxidative fibers (Gollnick et al., 1972). A recent study (Shaw et al., 2020) indeed showed that MFO rate was strongly related to the proportion of type I fibers in endurance and non-endurance trained males. Fat max $_{\max }$ (in $\mathrm{mL} \cdot \mathrm{kg}^{-1} \cdot \mathrm{min}^{-1}$ ) 
TABLE 2 | Whole-body fat oxidation parameters and SIN model variables.

\begin{tabular}{lcc}
\hline & Cyclists $(\boldsymbol{n}=\mathbf{1 1})$ & Active group $(\boldsymbol{n}=\mathbf{1 1})$ \\
\hline $\mathrm{MFO}\left[\mathrm{mg} \cdot \mathrm{kg}^{-1} \cdot \mathrm{min}^{-1}\right]$ & $6.5 \pm 1.5$ & $4.5 \pm 1.6^{\star}$ \\
$\mathrm{Fat}_{\max }\left[\mathrm{mL} \cdot \mathrm{kg}^{-1} \cdot \mathrm{min}^{-1}\right]$ & $33.9 \pm 6.6$ & $26.2 \pm 5.8^{\star}$ \\
$\mathrm{Fat}_{\text {max }}\left[\% \dot{\mathrm{V}} \mathrm{O}_{2 \text { peak }}\right]$ & $52.1 \pm 9.3$ & $53.2 \pm 8.3$ \\
Dilatation & $0.03 \pm 0.16$ & $0.25 \pm 0.15^{\star}$ \\
Symmetry & $1.36 \pm 0.49$ & $1.34 \pm 0.53$ \\
Translation & $0.18 \pm 0.22$ & $0.11 \pm 0.41$ \\
\hline
\end{tabular}

Data are presented as the mean $\pm S D$. MFO, maximal fat oxidation; Fat max,

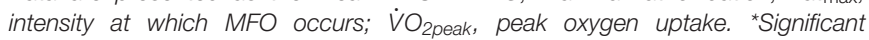
difference between groups ( $p \leq 0.05$ ).

TABLE 3 | Muscle deoxygenation kinetics parameters.

\begin{tabular}{lcc}
\hline & Cyclists $(\boldsymbol{n}=\mathbf{1 1})$ & Active group $(\boldsymbol{n}=\mathbf{1 1})$ \\
\hline$a_{1}$ & $2.39 \pm 0.39$ & $2.96 \pm 0.66^{\star}$ \\
$a_{2}$ & $-1.88 \pm 0.69$ & $-2.47 \pm 0.92$ \\
$a_{3}$ & $0.51 \pm 0.44$ & $0.49 \pm 0.44$ \\
$B$ & $-72.59 \pm 14.93$ & $-96.81 \pm 23.94^{\star}$ \\
{$[\mathrm{HHb}]_{\mathrm{BP}}\left[\mathrm{mL} \cdot \mathrm{kg}^{-1} \cdot \mathrm{min}^{-1}\right]$} & $44.8 \pm 3.1$ & $31.4 \pm 4.6^{\star}$ \\
{$[\mathrm{HHb}]_{\mathrm{BP}}\left[\% \dot{V} \mathrm{O}_{2 \text { peak }}\right]$} & $69.0 \pm 5.6$ & $64.0 \pm 7.7$
\end{tabular}

Data are presented as the mean $\pm S D$. $a_{1}$ and $a_{3}$, slopes of the first and second parts of the curve of the double linear model, respectively; $a_{2}$, change in the slope between the first and the second parts of the curve of the double linear model; $b, y$-intercept of the first part of the curve of the double linear model; $[\mathrm{HH} b]_{\mathrm{BP}}$, breakpoint of the curve of the double linear model; $\dot{V} O_{2 p e a k}$, peak oxygen uptake. *Significant difference between groups ( $p \leq 0.05)$.

was also higher in cyclists than in the active group (+29\%), whereas no difference was found for Fat ${ }_{\max }$ when expressed as relative intensity $\left(\% \dot{V} O_{2 p e a k}\right)$, corroborating previous findings (Achten and Jeukendrup, 2003; Lima-Silva et al., 2010). Unlike our hypothesis, in addition to similar symmetry and translation values, the dilatation variable was unexpectedly greater in the active group than in the cyclists (Figure $\mathbf{2 A}$ and Table 2). In previous studies using similar submaximal incremental protocols and data analysis with SIN, the dilatation values corresponded to $\sim 0.1$ in moderately trained men $\left(\dot{V} O_{2 p e a k}\right.$ of $\sim 50 \mathrm{~mL} \cdot \mathrm{kg}^{-1} \cdot \mathrm{min}^{-1}$ ) (Cheneviere et al., 2011, 2012), while the mean values in an obese population (BMI > 35) ranged between -0.1 and 0.4 (Lanzi et al., 2014, 2015). According to these results, and although an averaging process for the two submaximal tests has been used to reduce the intraindividual variability of fat oxidation rates (Croci et al., 2014), the dilatation value seems to be more individual-dependent than training status or body composition related. Inter-individual or between-group comparisons in terms of the global shape of fat oxidation kinetics may thus be questionable and require further research. Nevertheless, the assessment of the global shape of these kinetics seems to be more sensitive and adapted to put forward differences in longitudinal study designs (Lanzi et al., 2015). However, in the present study, trained cyclists exhibited greater fat oxidation rates with significant upward fat oxidation kinetics.
TABLE 4 | Correlations between fat oxidation kinetics and muscle deoxygenation kinetics parameters.

\begin{tabular}{|c|c|c|c|}
\hline \multirow[t]{2}{*}{$(n=22)$} & \multicolumn{3}{|c|}{$\% \Delta[\mathrm{HHb}]$} \\
\hline & $a_{1}$ & $a_{3}$ & {$[\mathrm{HHb}]_{\mathrm{BP}}\left[\mathrm{mL} \cdot \mathrm{kg}^{-1} \cdot \mathrm{min}^{-1}\right]$} \\
\hline MFO $\left[\mathrm{mg} \cdot \mathrm{kg}^{-1} \cdot \mathrm{min}^{-1}\right]$ & $-0.41^{\S}$ & & $0.66^{\star}$ \\
\hline Fat $_{\max }\left[\mathrm{mL} \cdot \mathrm{kg}^{-1} \cdot \mathrm{min}^{-1}\right]$ & $-0.41^{\S}$ & $0.47^{\star}$ & $0.68^{\star}$ \\
\hline Fatmax $_{\max }\left[\% \mathrm{VO}_{2 \text { peak }}\right]$ & & $0.52^{*}$ & \\
\hline Symmetry & & $0.58^{*}$ & \\
\hline
\end{tabular}

Data are presented as Pearson correlation coefficients ( $r$. . $a_{1}$ and $a_{3}$, slopes of the first and second parts of the curve of the double linear model, respectively; $[\mathrm{HHb}]_{\mathrm{BP}}$, breakpoint of the curve of the double linear model; $\Delta[\mathrm{HH}]$ ], variation in deoxygenated hemo- and myoglobin concentration; MFO, maximal fat oxidation; Fat ${ }_{\text {max }}$, intensity at which MFO occurs; $\dot{V} O_{2 p e a k}$, peak oxygen uptake. *Significant correlation ( $p \leq 0.05)$. ${ }^{\circledR} p=0.06$.

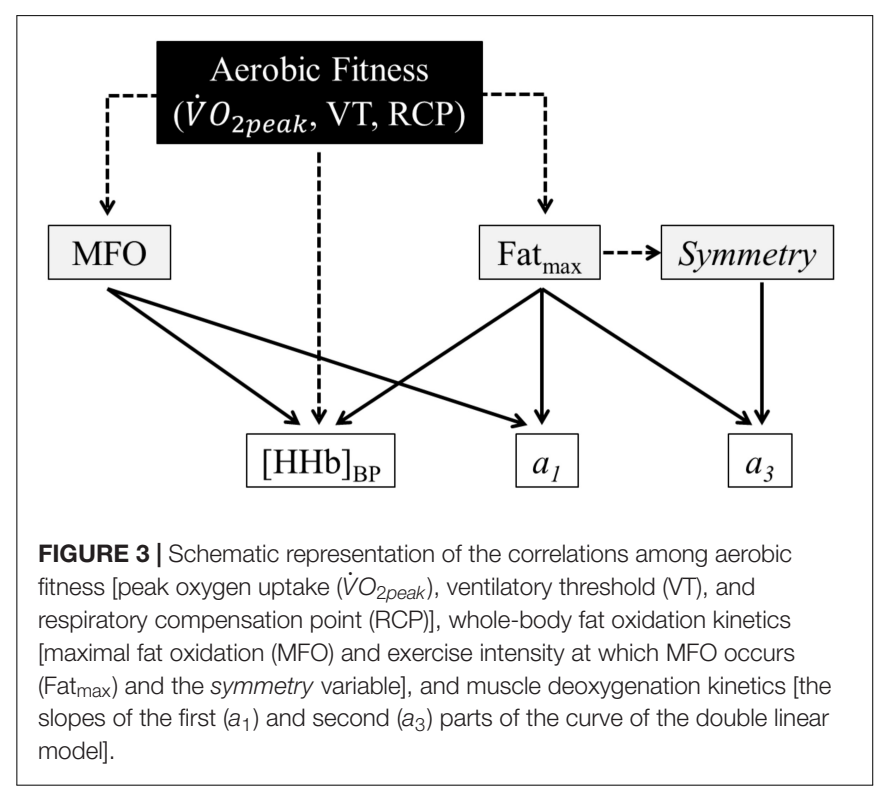

\section{Effect of Aerobic Fitness on Muscle Deoxygenation Kinetics}

In addition to greater fat oxidation, cyclists also showed a better balance between $\mathrm{O}_{2}$ delivery and consumption, reflected by a slower slope $\left(a_{1}\right)$ of $\% \Delta[\mathrm{HHb}]$ kinetics $(-20 \%)$ and a $[\mathrm{HHb}]_{\mathrm{BP}}$ occurring at a significantly higher absolute intensity (+42\%) compared with the active group (Table 3). This was in accordance with previous studies, which found a right-shifted $[\mathrm{HHb}]_{\mathrm{BP}}$ in individuals with greater $\dot{\mathrm{V}} \mathrm{O}_{2 \text { peak }}$ (Boone et al., 2009; Murias et al., 2013; McNarry et al., 2015; Boone et al., 2016a). The strong and positive correlations found between the absolute intensity at which the $[\mathrm{HHb}]_{\mathrm{BP}}$ occurred and $\dot{V} \mathrm{O}_{2 p e a k}$, MAP, VT, and RCP confirm the likely influence of aerobic fitness status on muscle deoxygenation kinetics. The shallower slope in cyclists indicates a lower reliance on $\mathrm{O}_{2}$ extraction from the blood flow by myocytes for a given $\dot{V} \mathrm{O}_{2}$, reflecting a delayed reliance on glycolytic metabolism (Boone et al., 2009, 2016a). This may be due to a greater proportion of slow oxidative fibers (Gollnick et al., 1972) and/or a higher muscle capillary network (i.e., higher capillaryto-myocyte ratio) inducing a greater muscle oxidative capacity 
in trained athletes (Boone et al., 2016a). However, we cannot confirm these morphological differences with our measurements.

It should be noted that $[\mathrm{HHb}]_{\mathrm{BP}}$ determined in the present study [69\% $\dot{V} O_{2 p e a k}$ (range: $61-76$ ) and 64\% $\dot{V} O_{2 p e a k}$ (range: 55-80) in cyclists and active individuals, respectively] occurred at lower relative exercise intensity found in previous studies. In fact, mean $[\mathrm{HHb}]_{\mathrm{BP}}$ values typically ranged from $\sim 75$ to $88 \%$ $\dot{V} O_{2 p e a k}$ in physically active male with heterogeneous aerobic fitness level (Bellotti et al., 2013; Murias et al., 2013; Fontana et al., 2015; Boone et al., 2016a). However, all these studies determined $[\mathrm{HHb}]_{\mathrm{BP}}$ during a single maximal incremental ramp test with 1min stage duration, contrasting with the submaximal incremental test and 5-min stages used in the present study. This stage duration was needed to properly determine fat oxidation rates by ensuring $\dot{\mathrm{V}} \mathrm{O}_{2}$ and $\dot{\mathrm{V}} \mathrm{CO}_{2}$ steady-state values in the last minute of each step of the submaximal incremental test in both active and cyclist participants (Amaro-Gahete et al., 2019). Simultaneously, this stage duration allows us to also have a steady-state for $\% \Delta[\mathrm{HHb}]$ at each step of the submaximal incremental test and, thus, appropriately determine the relationship between wholebody fat oxidation and muscle deoxygenation kinetics during the same exercise (Figure 4). Compared to previous studies, the longer stage duration of our incremental test may have induced a lower $\dot{V} \mathrm{O}_{2}$ and consequently lower $\% \dot{V} \mathrm{O}_{2 p e a k}$ for $[\mathrm{HHb}]_{\mathrm{BP}}$ of our participants as previously shown for RCP (Bourdon et al., 2018; Jamnick et al., 2018). These differences in test protocol/procedure make the comparison of results difficult and may explain the present lower relative $[\mathrm{HHb}]_{\mathrm{BP}}$ values compared to above studies. However, the fact that $[\mathrm{HHb}]_{\mathrm{BP}}$ occurred at a higher absolute exercise intensity in individuals with greater $\dot{V} O_{2 p e a k}$ is in accordance with previous results (Boone et al., 2009; Gravelle et al., 2012; Murias et al., 2013; McNarry et al., 2015; Boone et al., 2016a).

\section{Relationship Between Fat Oxidation and Muscle Deoxygenation Kinetics}

In the present study, whole-body fat oxidation and muscle deoxygenation kinetics are both influenced by aerobic fitness status and seem to be dependent on the balance between $\mathrm{O}_{2}$ delivery and consumption, inducing a relationship between the two kinetics. This was confirmed by different correlations among the parameters of the two kinetics (Table 4 and Figure 3). In fact, MFO and Fat max $_{\max }$ were positively correlated with the $[\mathrm{HHb}]_{\mathrm{BP}}$ and tended to be negatively correlated with $a_{1}$. This finding demonstrates that shallower muscle deoxygenation kinetics with a right-shifted $[\mathrm{HHb}]_{\mathrm{BP}}$ would be associated with greater fat oxidation rates. Indeed, the adaptations induced by endurance training, such as an increased proportion of slow oxidative fibers and a developed capillarization (Hawley et al., 2014), may be responsible for a longer maintained balance between $\mathrm{O}_{2}$ delivery and consumption during ramp exercise (Gollnick et al., 1972; Ferreira et al., 2007; Boone et al., 2009), which provides slower muscle deoxygenation at the same absolute exercise intensity and is associated with prolonged fat oxidation during high exercise intensities. Endurance training adaptations in cyclists promote a greater peripheral muscle capacity to extract and use $\mathrm{O}_{2}$ to oxidize fat during incremental exercise, shifting Fat max $_{\text {mo higher }}$ absolute exercise intensities compared with active individuals. In addition, the absolute and relative values of Fat max $_{\text {, a }}$ as well as the symmetry variable of the SIN model, were all positively correlated with the slope of the second part of the double linear model $\left(a_{3}\right)$. In other words, whole-body fat oxidation kinetics presenting a rightward asymmetry; thus, a right-shifted Fat max $_{\text {max }}$ may be associated with a steeper slope of the second part of the $\% \Delta[\mathrm{HHb}]$ kinetics. This may reflect a prolonged reliance on aerobic metabolism and thus a prolonged utilization of fat as an energetic substrate, even at high intensity when glycolytic metabolism provides more energy during exercise compared with lower intensities. In fact, our $a_{3}$ values are different from zero, attesting that, at the end of the submaximal incremental test, the upper limits of the fractional $\mathrm{O}_{2}$ extraction were not yet reached (the test ended at $87 \% \dot{V} O_{2 p e a k}$ ). These correlations confirmed our hypothesis that a relationship exists between whole-body fat oxidation and muscle deoxygenation kinetics.

\section{Methodological Limitations}

Our suggestions on the differences in fat oxidation and muscle deoxygenation between the two groups are based on
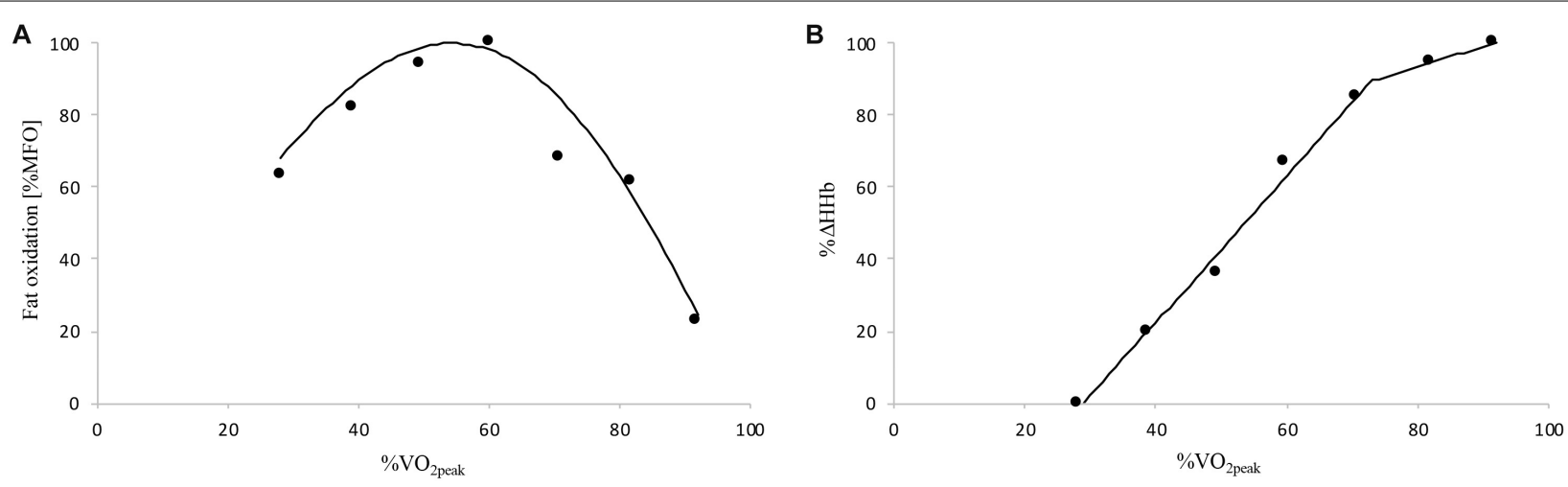

FIGURE 4 | Example of the whole-body fat oxidation (A) and muscle deoxygenation (B) kinetics of a cyclist participant determined during the submaximal incremental test. $\Delta[\mathrm{HHb}]$, variation in deoxygenated hemo- and myoglobin concentration; MFO, maximal fat oxidation; $\dot{\mathrm{V}} \mathrm{O}_{2 p e a k}$, peak oxygen uptake. 
possible muscular adaptations, such as differences in muscle fiber proportions. However, muscle biopsies were not taken in the present study, and we can only speculate about the likely relationship between the higher proportion of slow oxidative fibers and slower $\% \Delta[\mathrm{HHb}]$ kinetics and increased fat metabolism in cyclists compared with active individuals. Likewise, we did not measure oxidative enzyme activity, and we could thus not state whether cyclists had a greater fat oxidation because of an enhanced muscle enzyme activity (Stisen et al., 2006).

\section{CONCLUSION}

Our findings confirmed the influence of aerobic fitness status on both whole-body fat oxidation (Achten and Jeukendrup, 2003; Lima-Silva et al., 2010) and muscle deoxygenation kinetics (Boone et al., 2016a), and to the best of our knowledge, this was the first study to show a relationship between the two kinetics. Our results showed that shallower $\% \Delta[\mathrm{HHb}]$ kinetics with a right-shifted $[\mathrm{HHb}]_{\mathrm{BP}}$ were associated with higher fat oxidation (MFO) and $\mathrm{Fat}_{\max }$ shifted to higher absolute exercise intensities. This corroborates that, compared to active individuals, endurance-trained cyclists may maintain aerobic ATP production until higher exercise intensities and may have a delayed reliance on glycolytic metabolism because of a longer

\section{REFERENCES}

Achten, J., Gleeson, M., and Jeukendrup, A. E. (2002). Determination of the exercise intensity that elicits maximal fat oxidation. Med. Sci. Sports Exerc. 34, 92-97. doi: 10.1097/00005768-200201000-00015

Achten, J., and Jeukendrup, A. E. (2003). Maximal fat oxidation during exercise in trained men. Int. J. Sports Med. 24, 603-608. doi: 10.1055/s-2003-43265

Amaro-Gahete, F. J., Sanchez-Delgado, G., Jurado-Fasoli, L., De-la, O. A., Castillo, M. J., Helge, J. W., et al. (2019). Assessment of maximal fat oxidation during exercise: a systematic review. Scand. J. Med. Sci. Sports 29, 910-921. doi: 10. $1111 /$ sms. 13424

Beaver, W. L., Wasserman, K., and Whipp, B. J. (1986). A new method for detecting anaerobic threshold by gas exchange. J. Appl. Physiol. 60, 2020-2027. doi: 10.1152/jappl.1986.60.6.2020

Bellotti, C., Calabria, E., Capelli, C., and Pogliaghi, S. (2013). Determination of maximal lactate steady state in healthy adults: can NIRS help? Med. Sci. Sports Exerc. 45, 1208-1216. doi: 10.1249/MSS.0b013e3182828ab2

Bishop, D. J., Granata, C., and Eynon, N. (2014). Can we optimise the exercise training prescription to maximise improvements in mitochondria function and content? Biochim. Biophys. Acta 1840, 1266-1275. doi: 10.1016/j.bbagen.2013. 10.012

Boone, J., Barstow, T. J., Celie, B., Prieur, F., and Bourgois, J. (2016a). The interrelationship between muscle oxygenation, muscle activation, and pulmonary oxygen uptake to incremental ramp exercise: influence of aerobic fitness. Appl. Physiol. Nutr. Metab. 41, 55-62. doi: 10.1139/apnm-20150261

Boone, J., Bouckaert, J., Barstow, T. J., and Bourgois, J. (2012). Influence of priming exercise on muscle deoxy[Hb $+\mathrm{Mb}]$ during ramp cycle exercise. Eur. J. Appl. Physiol. 112, 1143-1152. doi: 10.1007/s00421-011-2068-z

Boone, J., Koppo, K., Barstow, T. J., and Bouckaert, J. (2009). Pattern of deoxy $[\mathrm{Hb}+\mathrm{Mb}]$ during ramp cycle exercise: influence of aerobic fitness status. Eur. J. Appl. Physiol. 105, 851-859. doi: 10.1007/s00421-008-0969-2

Boone, J., Vandekerckhove, K., Coomans, I., Prieur, F., and Bourgois, J. G. (2016b). An integrated view on the oxygenation responses to incremental exercise at maintained balance between $\mathrm{O}_{2}$ delivery and consumption, supporting higher fat oxidation rates.

\section{DATA AVAILABILITY STATEMENT}

The datasets generated for this study are available on request to the corresponding author.

\section{ETHICS STATEMENT}

The studies involving human participants were reviewed and approved by Commission cantonale d'Éthique de la Recherche sur l'être humain Vaud (CER-VD)-Swissethics. The patients/participants provided their written informed consent to participate in this study.

\section{AUTHOR CONTRIBUTIONS}

$\mathrm{AZ}, \mathrm{XC}$, and DM conceived and designed the research study. AZ, SL, CT, NB, XC, and DM performed the experiments. AZ, SL, LV, CT, XC, and DM analyzed the data. AZ, BG, BK, XC, and $\mathrm{DM}$ interpreted the results of the experiments. $\mathrm{AZ}, \mathrm{XC}$, and DM prepared figures and drafted the manuscript. All authors approved the final version of the manuscript.

the brain, the locomotor and respiratory muscles. Eur. J. Appl. Physiol. 116, 2085-2102. doi: 10.1007/s00421-016-3468-x

Bourdon, P. C., Woolford, S. M., and Buckley, J. D. (2018). Effects of varying the step duration on the determination of lactate thresholds in elite rowers. Int. J. Sports Physiol. Perform. 13, 687-693. doi: 10.1123/ijspp.2017-0258

Brooks, G. A., and Mercier, J. (1994). Balance of carbohydrate and lipid utilization during exercise: the "crossover" concept. J. Appl. Physiol. 76, 2253-2261. doi: 10.1152/jappl.1994.76.6.2253

Broxterman, R. M., Craig, J. C., and Richardson, R. S. (2018). The respiratory compensation point and the deoxygenation break point are not valid surrogates for critical power and maximum lactate steady state. Med. Sci. Sports Exerc. 50, 2379-2382. doi: 10.1249/MSS.0000000000001699

Cheneviere, X., Borrani, F., Droz, D., Gojanovic, B., and Malatesta, D. (2012). Effects of 2 different prior endurance exercises on whole-body fat oxidation kinetics: light vs. heavy exercise. Appl. Physiol. Nutr. Metab. 37, 955-964. doi: 10.1139/h2012-076

Cheneviere, X., Borrani, F., Sangsue, D., Gojanovic, B., and Malatesta, D. (2011). Gender differences in whole-body fat oxidation kinetics during exercise. Appl. Physiol. Nutr. Metab. 36, 88-95. doi: 10.1139/H10-086

Cheneviere, X., Malatesta, D., Peters, E. M., and Borrani, F. (2009). A mathematical model to describe fat oxidation kinetics during graded exercise. Med. Sci. Sports Exerc. 41, 1615-1625. doi: 10.1249/MSS.0b013e31819e2f91

Croci, I., Borrani, F., Byrne, N. M., Wood, R. E., Hickman, I. J., Cheneviere, X., et al. (2014). Reproducibility of Fatmax and fat oxidation rates during exercise in recreationally trained males. PLoS One 9:e97930. doi: 10.1371/journal.pone. 0097930

DeLorey, D. S., Kowalchuk, J. M., and Paterson, D. H. (2003). Relationship between pulmonary $\mathrm{O} 2$ uptake kinetics and muscle deoxygenation during moderate-intensity exercise. J. Appl. Physiol. (1985) 95, 113-120. doi: 10.1152/ japplphysiol.00956.2002

Duncan, A., Meek, J. H., Clemence, M., Elwell, C. E., Tyszczuk, L., Cope, M., et al. (1995). Optical pathlength measurements on adult head, calf and forearm and the head of the newborn infant using phase resolved optical spectroscopy. Phys. Med. Biol. 40, 295-304. doi: 10.1088/0031-9155/40/2/007 
Durnin, J. V., and Womersley, J. (1973). Total body fat, calculated from body density, and its relationship to skinfold thickness in 571 people aged 12-72 years. Proc. Nutr. Soc. 32:45A.

Ferrari, M., Binzoni, T., and Quaresima, V. (1997). Oxidative metabolism in muscle. Philos. Trans. R. Soc. Lond. B Biol. Sci. 352, 677-683. doi: 10.1098/rstb. 1997.0049

Ferreira, L. F., Koga, S., and Barstow, T. J. (2007). Dynamics of noninvasively estimated microvascular $\mathrm{O} 2$ extraction during ramp exercise. J.Appl. Physiol. (1985) 103, 1999-2004. doi: 10.1152/japplphysiol.01414.2006

Fontana, F. Y., Keir, D. A., Bellotti, C., De Roia, G. F., Murias, J. M., and Pogliaghi, S. (2015). Determination of respiratory point compensation in healthy adults: can non-invasive near-infrared spectroscopy help? J. Sci. Med. Sport 18, 590595. doi: 10.1016/j.jsams.2014.07.016

Frandsen, J., Vest, S. D., Larsen, S., Dela, F., and Helge, J. W. (2017). Maximal fat oxidation is related to performance in an ironman triathlon. Int. J. Sports Med. 38, 975-982. doi: 10.1055/s-0043-117178

Frayn, K. N. (1983). Calculation of substrate oxidation rates in vivo from gaseous exchange. J. Appl. Physiol. 55, 628-634. doi: 10.1152/jappl.1983.55.2.628

Gollnick, P. D., Armstrong, R. B., Saubert, C. W. T., Piehl, K., and Saltin, B. (1972). Enzyme activity and fiber composition in skeletal muscle of untrained and trained men. J. Appl. Physiol. 33, 312-319. doi: 10.1152/jappl.1972.33.3.312

Grassi, B., and Quaresima, V. (2016). Near-infrared spectroscopy and skeletal muscle oxidative function in vivo in health and disease: a review from an exercise physiology perspective. J. Biomed. Opt. 21:091313. doi: 10.1117/1.JBO. 21.9.091313

Gravelle, B. M., Murias, J. M., Spencer, M. D., Paterson, D. H., and Kowalchuk, J. M. (2012). Adjustments of pulmonary O2 uptake and muscle deoxygenation during ramp incremental exercise and constant-load moderate-intensity exercise in young and older adults. J. Appl. Physiol. (1985) 113, 1466-1475. doi: 10.1152/japplphysiol.00884.2011

Hawley, J. A., Hargreaves, M., Joyner, M. J., and Zierath, J. R. (2014). Integrative biology of exercise. Cell 159, 738-749. doi: 10.1016/j.cell.2014.10.029

Holloszy, J. O., and Coyle, E. F. (1984). Adaptations of skeletal muscle to endurance exercise and their metabolic consequences. J. Appl. Physiol. 56, 831-838. doi: 10.1152/jappl.1984.56.4.831

Jamnick, N. A., Botella, J., Pyne, D. B., and Bishop, D. J. (2018). Manipulating graded exercise test variables affects the validity of the lactate threshold and [Formula: see text]. PLoS One 13:e0199794. doi: 10.1371/journal.pone.0199794

Keir, D. A., Pogliaghi, S., and Murias, J. M. (2018). The respiratory compensation point and the deoxygenation break point are valid surrogates for critical power and maximum lactate steady state. Med. Sci. Sports Exerc. 50, 2375-2378. doi: 10.1249/MSS.0000000000001698

Koga, S., Rossiter, H. B., Heinonen, I., Musch, T. I., and Poole, D. C. (2014). Dynamic heterogeneity of exercising muscle blood flow and $\mathrm{O} 2$ utilization. Med. Sci. Sports Exerc. 46, 860-876. doi: 10.1249/MSS.0000000000000178

Lanzi, S., Codecasa, F., Cornacchia, M., Maestrini, S., Capodaglio, P., Brunani, A., et al. (2015). Short-term HIIT and Fat max training increase aerobic and metabolic fitness in men with class II and III obesity. Obesity (Silver Spring) 23, 1987-1994. doi: 10.1002/oby.21206

Lanzi, S., Codecasa, F., Cornacchia, M., Maestrini, S., Salvadori, A., Brunani, A., et al. (2014). Fat oxidation, hormonal and plasma metabolite kinetics during a submaximal incremental test in lean and obese adults. PLoS One 9:e88707. doi: 10.1371/journal.pone.0088707

Laplaud, D., Hug, F., and Grelot, L. (2006). Reproducibility of eight lower limb muscles activity level in the course of an incremental pedaling exercise. J. Electromyogr. Kinesiol. 16, 158-166. doi: 10.1016/j.jelekin.2005.04.002

Lima-Silva, A. E., Bertuzzi, R. C., Pires, F. O., Gagliardi, J. F., Barros, R. V., Hammond, J., et al. (2010). Relationship between training status and maximal fat oxidation rate. J. Sports Sci. Med. 9, 31-35.

Malatesta, D., Werlen, C., Bulfaro, S., Cheneviere, X., and Borrani, F. (2009). Effect of high-intensity interval exercise on lipid oxidation during postexercise recovery. Med. Sci. Sports Exerc. 41, 364-374. doi: 10.1249/MSS. ob013e3181857edo

McNarry, M. A., Farr, C., Middlebrooke, A., Welford, D., Breese, B., Armstrong, N., et al. (2015). Aerobic function and muscle deoxygenation dynamics during ramp exercise in children. Med. Sci. Sports Exerc. 47, 1877-1884. doi: 10.1249/ MSS.0000000000000609

Murias, J. M., Keir, D. A., Spencer, M. D., and Paterson, D. H. (2013). Sex-related differences in muscle deoxygenation during ramp incremental exercise. Respir. Physiol. Neurobiol. 189, 530-536. doi: 10.1016/j.resp.2013.08.011

Nordby, P., Saltin, B., and Helge, J. W. (2006). Whole-body fat oxidation determined by graded exercise and indirect calorimetry: a role for muscle oxidative capacity? Scand. J. Med. Sci. Sports 16, 209-214. doi: 10.1111/j.16000838.2005.00480.x

Sahlin, K., Sallstedt, E. K., Bishop, D., and Tonkonogi, M. (2008). Turning down lipid oxidation during heavy exercise-what is the mechanism? J. Physiol. Pharmacol. 59(Suppl. 7), 19-30.

Shaw, C. S., Swinton, C., Morales-Scholz, M. G., McRae, N. L., Erftemeyer, T., Aldous, A., et al. (2020). The impact of exercise training status on the fibre type specific abundance of proteins regulating intramuscular lipid metabolism. J. Appl. Physiol. (1985) 128, 379-389. doi: 10.1152/japplphysiol.00797.2019

Spencer, M. D., Murias, J. M., and Paterson, D. H. (2012). Characterizing the profile of muscle deoxygenation during ramp incremental exercise in young men. Eur. J. Appl. Physiol. 112, 3349-3360. doi: 10.1007/s00421-012-2323-y

Stisen, A. B., Stougaard, O., Langfort, J., Helge, J. W., Sahlin, K., and Madsen, K. (2006). Maximal fat oxidation rates in endurance trained and untrained women. Eur. J. Appl. Physiol. 98, 497-506. doi: 10.1007/s00421-006-0290-x

Wasserman, K., Whipp, B. J., Koyl, S. N., and Beaver, W. L. (1973). Anaerobic threshold and respiratory gas exchange during exercise. J. Appl. Physiol. 35, 236-243. doi: 10.1152/jappl.1973.35.2.236

Conflict of Interest: The authors declare that the research was conducted in the absence of any commercial or financial relationships that could be construed as a potential conflict of interest.

Copyright (C) 2020 Zurbuchen, Lanzi, Voirol, Trindade, Gojanovic, Kayser, Bourdillon, Chenevière and Malatesta. This is an open-access article distributed under the terms of the Creative Commons Attribution License (CC BY). The use, distribution or reproduction in other forums is permitted, provided the original author(s) and the copyright owner(s) are credited and that the original publication in this journal is cited, in accordance with accepted academic practice. No use, distribution or reproduction is permitted which does not comply with these terms. 